hep-th/0401084

January, 2004

\title{
BPS Domain Walls in Massive Hyper-Kähler Sigma Models
}

\author{
Masato Arai ${ }^{a *}$, Muneto Nitta ${ }^{b \dagger}$, and Norisuke Sakai ${ }^{c} \ddagger$ \\ ${ }^{a}$ Institute of Physics, AS CR, 182 21, Praha 8, Czech Republic \\ ${ }^{b}$ Department of Physics, Purdue University, West Lafayette, IN 47907-1396, USA \\ and \\ ${ }^{c}$ Department of Physics, Tokyo Institute of Technology \\ Tokyo 152-8551, JAPAN
}

\begin{abstract}
We discuss hypermultiplets admitting degenerate discrete vacua and BPS domain walls interpolating them. This talk is based on the original papers, hep-th/0307274, hep-th/0211103 and hep-th/0302028.
\end{abstract}

\footnotetext{
*e-mail address: arai@fzu.cz

$\dagger$ †-mail address: nitta@th.phys.titech.ac.jp

Address after December 11: Tokyo Institute of Technology

†e-mail address: nsakai@th.phys.titech.ac.jp
} 


\section{Introduction}

There exists a long history for searching topological defects such as domain walls. Lots of their properties in particular localization of massless or light fields on them have been investigated. Recent interest in the brane-world scenario [3] has brought us an idea to realize our world as the effective field theory of light localized fields on the brane. Supersymmetry (SUSY) is combined with this idea by considering the BPS solitons in SUSY field theories. They typically break the half of the original SUSY spontaneously. BPS domain walls are investigated in detail in $D=4, \mathcal{N}=1$ SUSY theories (with four supercharges), on which effective field theories have two unbroken supercharges. However in order to realize $D=4, \mathcal{N}=1$ SUSY theory on the world-volume, we need a higher-dimensional theory with eight supercharges. In these theories scalar multiplets are hypermultiplets. They must parameterize curved hyper-Kähler (HK) manifolds 4, 5] with a scalar potential admitting at least two discrete degenerate vacua. These models are called the massive HK nonlinear sigma models (NLSM).

In this talk, the HK quotient method [6, 7] to construct HK manifolds is shown to be generalized to the massive models and the BPS domain wall in the simplest case of the Eguchi-Hanson target space is given. Keeping essential properties of eight supercharges, we discuss a simpler and familiar case of $D=4, \mathcal{N}=2$ SUSY theories. Also we use the $\mathcal{N}=1$ superspace formalism. A fully off-shell $\mathcal{N}=2$ superspace (the Harmonic superspace) formalism is discussed in the original paper [1].

\section{Kähler Sigma Models and Walls}

Here we recall BPS walls in $\mathcal{N}=1$ SUSY theories, because we formulate $\mathcal{N}=2$ SUSY models in terms of $\mathcal{N}=1$ superfields in the following sections. Scalar fields belong to chiral superfields $\Phi^{i}(y, \theta)=\phi^{i}(y)+\sqrt{2} \theta \psi^{i}(y)+\theta \theta F^{i}(y)$ with $y^{\mu}=x^{\mu}+i \theta \sigma^{\mu} \bar{\theta}$. The Lagrangian for the most general $\mathcal{N}=1$ SUSY Lagrangian for chiral superfields (the generalized Wess-Zumino model) is given by 8

$$
\mathcal{L}=\int d^{4} \theta K\left(\Phi, \Phi^{\dagger}\right)+\left[\int d^{2} \theta W(\Phi)+\text { c.c. }\right]
$$


with $K$ and $W$ real and holomorphic functions, called the Kähler potential and the superpotential, respectively. After elimination of auxiliary fields by their equations of motion $F^{i}=-g^{i j^{*}} \partial_{j^{*}} W^{*}+$ fermions, the bosonic part is calculated as

$$
\mathcal{L}_{\text {boson }}=-g_{i j^{*}} \partial_{\mu} \phi^{i} \partial^{\mu} \phi^{* j}-g^{i j^{*}} \partial_{i} W \partial_{j^{*}} W^{*}
$$

with $g_{i j^{*}} \equiv \partial_{i} \partial_{j^{*}} K$ the Kähler metric. (We denote $\partial_{i}=\frac{\partial}{\partial \phi^{2}}$.) The target manifold must be a Kähler manifold and therefore these models are called Kähler sigma models.

Assuming a domain wall configuration perpendicular to the third axis $x^{3}=z$, its energy density per unit area in the $x-y$ plane is given by

$$
\begin{aligned}
E & =\int d z\left(g_{i j^{*}} \partial_{z} \phi^{i} \partial_{z} \phi^{* j}+g^{i j^{*}} \partial_{i} W \partial_{j^{*}} W^{*}\right) \\
& =\int d z\left|\partial_{z} \phi^{i}-e^{i \alpha} g^{i k^{*}} \partial_{k^{*}} W^{*}\right|^{2}+\int d z\left(e^{i \alpha} \partial_{z} \phi^{i} \partial_{i} W+\text { c.c. }\right) \\
& \geq \int d z\left(\partial_{z} \phi^{i} \partial_{i} W+\text { c.c. }\right)=2 \operatorname{Re}\left(e^{i \alpha} \Delta W\right)
\end{aligned}
$$

with the norm defined by $\left|V^{i}\right|^{2} \equiv g_{i j^{*}} V^{i} V^{* j},\left.\Delta W \equiv W\right|_{z=\infty}-\left.W\right|_{z=-\infty}$ and $\alpha$ arbitrary real constant. Since we obtain the best bound at $e^{-i \alpha}=\Delta W /|\Delta W|$, we derive the BPS bound $E \geq 2|\Delta W|$ saturated by solutions of the BPS equation

$$
\partial_{z} \phi^{i}=e^{-i \alpha} g^{i j^{*}} \partial_{j^{*}} W^{*}, \quad e^{-i \alpha}=\Delta W /|\Delta W|
$$

The SUSY transformation on the fermion $\psi^{i}$ with a BPS wall background is calculated as $\delta_{\epsilon} \psi^{i}=$ $\sqrt{2}\left(i \sigma^{\mu} \bar{\epsilon} \partial_{\mu} \phi^{i}+\epsilon F^{i}\right)=\sqrt{2}\left(i \sigma^{z} \bar{\epsilon} e^{-i \alpha}-\epsilon\right) g^{i j^{*}} \partial_{j^{*}} W^{*}$. Therefore two SUSYs satisfying $i e^{-i \alpha} \sigma^{z} \bar{\epsilon}=\epsilon$ out of four are preserved and so the solutions are called 1/2 BPS states.

The BPS domain walls (and their junction) in $D=4, \mathcal{N}=1$ SUSY NLSMs including runaway vacua and singularity of the metric were discussed [9]. It is easy to find $\mathcal{N}=1$ SUSY models admitting wall solutions, because $K$ and $W$ are arbitrary and independent to each other, but they are not for $\mathcal{N}=2$ SUSY as we will see in the following sections. 


\section{Massive Hyper-Kähler Sigma Models and Walls}

We discuss hypermultiplets with potential terms. The on-shell component Lagrangian for massive HK model is well known [5], whose bosonic part is

$$
\mathcal{L}_{\text {boson }}=-g_{i j^{*}} \partial_{\mu} \phi^{i} \partial^{\mu} \phi^{* j}-|\mu|^{2} g_{i j^{*}} k^{i} k^{* j}
$$

with $g_{i j^{*}}$ the target HK metric, $\mu$ a complex mass parameter and $k^{i}\left(\phi, \phi^{*}\right)$ a tri-holomorphic Killing vector on the target HK manifold. ${ }^{1}$ Therefore an isometry on the manifold is required for a nontrivial potential to exist and vacua are given by fixed points of its action.

We take $\mu$ real because phase can be absorbed into the definition of $k^{i}$. Energy density for a wall perpendicular to the $z$-axis is

$$
\begin{aligned}
E & =\int d z\left(g_{i j^{*}} \partial_{z} \phi^{i} \partial_{z} \phi^{* j}+\mu^{2} g_{i j^{*}} k^{i} k^{* j}\right) \\
& =\int d z\left|\partial_{z} \phi^{i}-\mu k^{i}\right|^{2}+\int d z\left(\mu g_{i j^{*}} k^{i} \partial_{z} \phi^{* j}+\text { c.c. }\right) \\
& \geq \int d z\left(\mu g_{i j^{*}} k^{i} \partial_{z} \phi^{* j}+\text { c.c. }\right)=\mu \Delta D
\end{aligned}
$$

where $k^{i}=g^{i j^{*}} \partial_{j^{*}} D$ with $D\left(\phi, \phi^{*}\right)$ a real function called the Killing potential (moment map) ${ }^{2}$ and $\left.\Delta D \equiv D\right|_{z=\infty}-\left.D\right|_{z=-\infty}$. We thus obtain the BPS bound $E \geq \mu \Delta D$ saturated by the BPS equation $\partial_{z} \phi^{i}=\mu k^{i}[11$.

The Eguchi-Hanson space $T^{*} \mathbf{C} P^{1}$ admits $S U(2)$ tri-holomorphic isometry, one of whose three generators, say $\sigma_{3}$, can be used to obtain the potential. A rotation around the third axis on the base $S^{2} \simeq \mathbf{C} P^{1}$ has two fixed points on the North and South poles, both of which are vacua. The BPS domain wall interpolating these vacua was firstly obtained by Abraham and Townsend [1] in the component formalism. Lots of interesting BPS solitons were constructed in toric HK manifolds [12, 13].

\footnotetext{
${ }^{1}$ The potential term can be interpreted by the Sherk-Schwarz (SS) dimensional reduction [10] from sixdimensions, where HK sigma models are massless. Then the SS reduction to four (five) space-time dimensions is defined by $-i\left(\partial_{5}+i \partial_{6}\right) \phi^{i}=\mu k^{i}$ with $\mu \in \mathbf{C}\left(\partial_{6} \phi^{i}=\mu k^{i}\right.$ with $\left.\mu \in \mathbf{R}\right)$.

${ }^{2}$ It is in general difficult to find $D$ for given manifold and Killing vector.
} 


\section{Massive Hyper-Kähler Models from $\mathcal{N}=2$ SUSY QCD}

Let $(\Phi, \Psi)$ be $\mathcal{N}=2$ hypermultiplets with $\Phi$ and $\Psi$ being $N \times M$ and $M \times N$ matrix chiral superfields. To obtain nontrivial vacua we need an $U(M)$ gauge symmetry introducing $\mathcal{N}=2$ vector multiplets $(V, \Sigma)$ with $V$ an $M \times M$ matrix vector superfield and $\Sigma$ an $M \times M$ matrix chiral superfield. We work out for the $U(M)$ gauge group in which $U(1)$ part is essential to obtain discrete vacua. We consider the Higgs branch of the theory taking the strong coupling limit $g \rightarrow \infty$ of gauge interactions, which eliminates the kinetic terms for $V$ and $\Sigma$. The gauge invariant Lagrangian is given by

$$
\begin{aligned}
\mathcal{L} & =\int d^{4} \theta\left[\operatorname{tr}\left(\Phi^{\dagger} \Phi e^{V}\right)+\operatorname{tr}\left(\Psi \Psi^{\dagger} e^{-V}\right)-c \operatorname{tr} V\right] \\
& +\left[\int d^{2} \theta\left\{\operatorname{tr}\left\{\Sigma\left(\Psi \Phi-b \mathbf{1}_{M}\right)\right\}+\sum_{a=1}^{N-1} m_{a} \operatorname{tr}\left(\Psi H_{a} \Phi\right)\right\}+\text { c.c. }\right],
\end{aligned}
$$

with $b \in \mathbf{C}$ and $c \in \mathbf{R}$ constituting a triplet of the Fayet-Iliopoulos parameters, $m_{a}$ complex mass and $H_{a}$ Cartan generators of $S U(N) \cdot{ }^{3}$ Eliminating superfields $V$ and $\Sigma$ using their algebraic equations of motion, we obtain the Lagrangian in terms of independent superfields, in which the Kähler potential is

$$
\begin{aligned}
K= & c \operatorname{tr} \sqrt{\mathbf{1}_{M}+\frac{4}{c^{2}} \Phi^{\dagger} \Phi \Psi \Psi^{\dagger}}-c \operatorname{tr} \log \left(\mathbf{1}_{M}+\sqrt{\mathbf{1}_{M}+\frac{4}{c^{2}} \Phi^{\dagger} \Phi \Psi \Psi^{\dagger}}\right) \\
& +c \operatorname{tr} \log \Phi^{\dagger} \Phi
\end{aligned}
$$

with a gauge fixing ${ }^{4}$

$$
\Phi=\left(\begin{array}{c}
\mathbf{1}_{M} \\
\varphi
\end{array}\right) Q, \quad \Psi=Q\left(\mathbf{1}_{M}, \psi\right), \quad Q=\sqrt{b}\left(\mathbf{1}_{M}+\psi \varphi\right)^{-\frac{1}{2}}
$$

with $\varphi(\psi)$ an $(N-M) \times M[M \times(N-M)]$ matrix chiral superfield, and the superpotential is

$$
W=b \sum_{a} m_{a} \operatorname{tr}\left[H_{a}\left(\begin{array}{c}
\mathbf{1}_{M} \\
\varphi
\end{array}\right)\left(\mathbf{1}_{M}+\psi \varphi\right)^{-1}\left(\mathbf{1}_{M}, \psi\right)\right] .
$$

\footnotetext{
${ }^{3}$ Flavor symmetry $\Phi \rightarrow \Phi^{\prime}=g \Phi, \Psi \rightarrow \Psi^{\prime}=\Psi g^{-1}$ with $g \in S U(N)$ in the massless limit $m_{a}=0$ is explicitly broken by the mass to its Cartan $U(1)^{N-1}$ generated by $H_{a}$.

${ }^{4}$ We discuss the $b \neq 0$ case here. The $b=0$ case must be discussed independently [1].
} 
This is the massive extension of the HK NLSM on the cotangent bundle over the Grassmann manifold, $T^{*} G_{N, M}$, found by Lindström and Roček [6]. This model contains ${ }_{N} C_{M}=N ! / M !(N-$ $M)$ ! discrete degenerate vacua corresponding to independent gauge fixing conditions (4.3) [1].

\section{Domain Wall Solutions}

As seen in Section 2, the tension of the BPS domain wall is given by superpotential. This fact implies that we should take $b \neq 0$ in $\mathcal{N}=1$ superfields. The $M=1$ case of $U(1)$ gauge symmetry reduces to $T^{*} \mathbf{C} P^{N-1}$ with the superpotential, which admits $N$ parallel domain walls [13]. Moreover if we take $N=2$ and $M=1$, the target space $T^{*} \mathbf{C} P^{1}$ is the Eguchi-Hanson space with the superpotential $W=b \frac{\mu}{1+\varphi \psi}\left(\mu \equiv m_{1}\right)$. The BPS eq. (2.4) in $\mathcal{N}=1$ superfields can be solved to give [2]

$$
\varphi=\psi^{*}=e^{|\mu|\left(z-z_{0}\right)} e^{i \delta}
$$

where $z_{0}$ and $\delta$ are integral constants. They correspond to zero modes arising from spontaneously broken translational invariance perpendicular to domain wall configuration and $U(1)$ isometry $\sigma_{3}$ in the internal space.

\section{Conclusions}

We have constructed massive HK NLSM on the cotangent bundle over $G_{N, M}$ in $\mathcal{N}=1$ superfields, which is the massive extension of Lindström and Roček. This model contains ${ }_{N} C_{M}=N ! / M !(N-$ $M)$ ! discrete degenerate vacua. A BPS Domain wall solution in the simplest $T^{*} \mathbf{C} P^{1}$ has been given.

Constructing domain walls in non-Abelian gauge group remains as an interesting future work. Turning on the gauge coupling does not change vacua. BPS walls should also be similar as shown in the $M=1$ case [13, 14]. Coupling to supergravity is possible as in the $M=1$ case [15]. 


\section{Acknowledgments}

We would like to thank Masashi Naganuma for discussions in the early stage of this work. M. N. is grateful to the organizers in QTS3 and the Argonne brane workshop. This work is supported in part by Grant-in-Aid for Scientific Research from the Japan Ministry of Education, Science and Culture 13640269 (NS). The work of M. N. was supported by the U. S. Department of Energy under grant DE-FG02-91ER40681 (Task B).

\section{References}

[1] M. Arai, M. Nitta and N. Sakai, hep-th/0307274.

[2] M. Arai, M. Naganuma, M. Nitta and N. Sakai, Nucl. Phys. B652 (2003) 35, hep-th/0211103; in Garden of Quanta - In honor of Hiroshi Ezawa, Eds. by J. Arafune et al. (World Scientific Publishing Co. Pte. Ltd. Singapore, 2003) pp 299-325, hep-th/0302028.

[3] N. Arkani-Hamed, S. Dimopoulos and G. Dvali, Phys. Lett. 429B (1998) 263, hep-ph/9803315. I. Antoniadis, N. Arkani-Hamed, S. Dimopoulos and G. Dvali, Phys. Lett. 436B (1998) 257, hep-ph/9804398. L. Randall and R. Sundrum, Phys. Rev. Lett. 83 (1999) 3370, hep-ph/9905221; Phys. Rev. Lett. 83 (1999) 4690, hep-th/9906064.

[4] L. Alvarez-Gaumé and D. Z. Freedman, Comm. Math. Phys. 80 (1981) 443.

[5] L. Alvarez-Gaumé and D. Z. Freedman, Comm. Math. Phys. 91 (1983) 87.

[6] U. Lindström and M. Roček, Nucl. Phys. B222 (1983) 285.

[7] N. J. Hitchin, A. Karlhede, U. Lindström and M. Roček, Comm. Math. Phys. 108 (1987) 535.

[8] J. Wess and J. Bagger, "Supersymmetry and Supergravity (2nd Ed.)", (1991), Princeton University Press. 
[9] M. Naganuma, M. Nitta and N. Sakai, Phys. Rev. D65 (2001) 045016, hep-th/0108179; in the Proceedings of the third international Sakharov conference on Physics Vol.2, (World Scientific Pub., 2003) pp.535-549, hep-th/0210205.

[10] J. Scherk and J. H. Schwarz, Phys. Lett. B82 (1979) 60.

[11] E. Abraham and P. K. Townsend, Phys. Lett. B 291 (1992) 85.

[12] J. P. Gauntlett, R. Portugues, D. Tong and P.K. Townsend, Phys. Rev. D63 (2001) 085002, hep-th/0008221. J. P. Gauntlett, D. Tong and P.K. Townsend, Phys. Rev. D63 (2001) 085001, hep-th/0007124; Phys. Rev. D64 (2001) 025010, hep-th/0012178. M. Naganuma, M. Nitta and N. Sakai, Grav. Cosmol. 8 (2002) 129, hep-th/0108133. R. Portugues and P. K. Townsend, JHEP 0204 (2002) 039, hep-th/0203181. M. Arai, E. Ivanov and J. Niederle, to be published in Nucl. Phys. B, hep-th/0312037.

[13] D. Tong, Phys. Rev. D66 (2002) 025013, hep-th/0202012. K. S. M. Lee, Phys. Rev. D67 (2003) 045009, hep-th/0211058.

[14] K. Kakimoto and N. Sakai, Phys. Rev. D68 (2003) 065005, hep-th/0306077. Y. Isozumi, K. Ohashi and N. Sakai, JHEP 0311 (2003) 061, hep-th/0310130; JHEP 0311 (2003) 060, hep-th/0310189.

[15] M. Arai, S. Fujita, M. Naganuma and N. Sakai, Phys. Lett. B556 (2003) 192, hep-th/0212175; hep-th/0311210. M. Eto, S. Fujita, M. Naganuma and N. Sakai, to be published in Phys. Rev. D, hep-th/0306198. 